\title{
The emotional and physical impact of wet age- related macular degeneration: findings from the wAMD Patient and Caregiver Survey
}

This article was published in the following Dove Press journal:

Clinical Ophthalmology

3 February 2016

Number of times this article has been viewed

\author{
Monica Varano' \\ Nicole Eter ${ }^{2}$ \\ Steve Winyard ${ }^{3}$ \\ Kim UWittrup-Jensen ${ }^{4}$ \\ Rafael Navarro ${ }^{5}$ \\ Julie Heraghty ${ }^{6}$ \\ On behalf of the wAMD \\ Patient and Caregiver \\ Survey Committee \\ members \\ 'Department of Ophthalmology, \\ Fondazione GB Bietti-IRCCS, Rome, \\ Italy; ${ }^{2}$ Department of Ophthalmology, \\ University of Münster, Münster, \\ Germany; ${ }^{3}$ Department of Policy \\ and Campaigns, Royal National \\ Institute of Blind People, London, UK; \\ ${ }^{4}$ Bayer Pharma AG, Berlin, Germany; \\ ${ }^{5}$ Instituto de Microcirugia Ocular, \\ Barcelona, Spain; ${ }^{\circ}$ Macular Disease \\ Foundation Australia, Sydney, NSW, \\ Australia
}

Objectives: This was a cross-sectional survey to evaluate the physical and emotional impact of wet age-related macular degeneration (wAMD) on a global cohort of patients who were receiving (or had previously received) antivascular endothelial growth factor injections, and caregivers (paid and unpaid).

Methods: The survey was performed in nine countries using an ophthalmologist-devised questionnaire.

Results: A total of 910 patients and 890 caregivers completed the questionnaire. Most patients had been diagnosed and receiving antivascular endothelial growth factor injections for more than 1 year $(74.7 \%$ and $63.8 \%$, respectively), and many patients $(82.1 \%)$ received support from a caregiver (usually a child/grandchild [47.3\%] or partner [23.3\%]). wAMD had a negative impact on most patients (71.6\%); many rated fear (44.9\%), sadness (39.9\%), frustration (37.3\%), and depression $(34.0 \%)$ as common. It was linked to physical consequences, such as difficulty in reading $(61.1 \%)$. Many effects were significantly greater in patients with a longer duration of disease or with wAMD in both eyes. Some caregivers (unpaid) also reported that caregiving had a negative impact on them (31.1\%); many reported emotions such as sadness $(34.9 \%)$ and depression (24.4\%), but many also felt useful (48.4\%). Overall, $27.2 \%$ of caregivers (unpaid) rated caregiving as inconvenient; this was linked to days of employment/personal obligations missed.

Conclusion: wAMD has a significant negative impact on the lives of patients, including vision-related depression, poor mobility, and limitations in day-to-day activities. The impact on nonprofessional caregivers may be underestimated in terms of emotional impact (such as depression) and loss of productivity.

Keywords: wet age-related macular degeneration, anti-vascular endothelial growth factor agents, wAMD Patient and Caregiver Survey, patient-reported outcomes

\section{Introduction}

It is estimated that there will be nearly 300 million people globally with age-related macular degeneration (AMD) by 2040. ${ }^{1}$ AMD is a progressive disease, and this growth level will be associated with a major economic burden, particularly in aging populations. From a societal perspective, AMD has been shown to adversely affect patient quality of life. Although much of the available evidence is based on small observational studies, these have clearly demonstrated an association between AMD and depression, poor mobility, and limited social functioning in older patients. ${ }^{2-7}$ There are fewer studies exploring the effects of wet age-related macular degeneration (wAMD) on caregivers. Surveys of caregivers of patients with chronic diseases indicate that inadequate income,
Correspondence: Monica Varano Department of Ophthalmology, Fondazione GB Bietti-IRCCS,

Via Livenza 3, Rome 00198, Italy

Tel +390685356727

Fax +390684242333

Email m.varano@mclink.it 
lack of respite or support, and level of caregiving tasks were associated with more negative outcomes, but these associations were dependent on caregiver-recipient relationship and type of illness. 8,9

A greater understanding of the emotional and physical impact of wAMD and the barriers to effective treatment from a patient and caregiver perspective may help to improve longterm management, improve treatment access and compliance, and address the wider quality-of-life issues associated with macular degeneration. At present, few studies have examined these factors on a more global scale and from the perspective of both the patient and caregiver.

The aim of this noninterventional, cross-sectional survey was to evaluate the impact of wAMD on a global cohort of patients who were currently receiving (or had previously received) antivascular endothelial growth factor (anti-VEGF) injections. This population was chosen as the authors were most interested in improving the management and well-being of wAMD patients treated with anti-VEGF agents, and the burden of treatment for wAMD patients and their caregivers was greater than for atrophic AMD. The survey also identified caregivers (both paid and unpaid), and evaluated the effect that caring for someone with wAMD had on unpaid caregivers. The survey was conducted via an ophthalmologist-devised questionnaire. A study that used a reconfigured version of this questionnaire, which was completed by 500 caregivers from Australia, has already been published. ${ }^{10}$ This 29 -item, selfadministered questionnaire used logistic regression analysis to assess the impact of caregiving on several domains, including psychological well-being, social activities, and retirement plans. This study showed that if the wAMD patient was highly dependent on the caregiver, this increased the odds of caregiver distress (odds ratio $=1.99$ ). More than half of the caregivers reported a negative state of mind and had to make changes to everyday activities and retirement plans as a result of caregiving. Distress was linked to level of sight loss experienced by the care recipient and health of caregiver $(69 \%$ of caregivers also reported health problems). However, this study did not focus on economic burden or loss of productivity associated with caregiving. These issues are assessed in this larger survey, which includes both caregivers and their patients. This survey will make an important contribution for assessing the impact of caring on caregivers.

\section{Methods Design}

This was a global, noninterventional, cross-sectional survey (questionnaire) of patients with wAMD and their caregivers that was conducted in nine countries (Australia, Brazil, Canada, France, Germany, Italy, Japan, Spain, and the UK) from June 2012 to September 2012 (including data analysis from July 2012 to December 2012). The questionnaire was devised by an independent steering committee consisting of ophthalmologists and experts, and the research organizations (Blueprint Partnership, Manchester, UK, and Survey Sampling International [SSI], London, UK). The questionnaire was designed to evaluate the emotional and physical impact of wAMD in patients and caregivers and also to identify barriers to treatment from their perspectives. Approval was obtained from the British Healthcare Business Intelligence Association (BHBIA). All materials had to be compliant with BHBIA's Legal and Ethical Guidelines for Healthcare Market Research. All participants provided informed consent.

The questionnaire was translated for each participating country, and was available via an online link. This link was soft-launched (ie, a small number of responders completed the questionnaire enabling a data check to ensure it was captured accurately). For those responders with poor eyesight, face-to-face and telephone collection methods were used, wherein a member of SSI or one of their online partners would read aloud the translated questions and collect and input the answers; all responses were anonymous.

\section{Recruitment}

Patients with an existing wAMD diagnosis (made by their own physician) and who received current or prior anti-VEGF injections (by their own physician) were included. Caregivers who provided care and support to a patient with wAMD (as described earlier) were also included. Support was defined as assisting with daily activities, being actively involved in clinical appointments, and/or influencing treatment decisions (eg, advising the patient). Patients and caregivers fulfilling the aforementioned criteria were enrolled using online recruitment via the SSI website, which was advertised through usual patient recruitment channels (eg, hospitals, clinics, care groups, etc). The research organization (SSI) also accessed physician databases, and those physicians who agreed to be involved referred patients (fulfilling the aforementioned criteria and with their consent) to the research organization. These patients also referred their caregivers.

\section{Data collection and analysis}

The questionnaire was divided into patient and caregiver sections. Each responder was asked to provide yes/no/not sure answers based on a number of available options, or to rate questions using impact scales (positive impact, no 
impact, negative impact), dependency scales (not dependent, neither dependent nor independent, dependent), or convenience scales (not inconvenient, neither convenient nor inconvenient, inconvenient). Professional caregivers did not participate in any questions related to the emotional impact of wAMD. It was assumed that if a caregiver was paid, then he or she will be less likely to be emotionally impacted by their client's eye disease.

Data checks were undertaken to ensure that all responders met the screening criteria. Responders who clicked through the survey in less than 2.5 minutes were removed, and responders who clicked through and only gave limited feedback (eg, only selecting the top option) or gave contradictory responses were removed.

All data were presented as descriptive statistics based on absolute percentages and means. Where possible, data were stratified according to length of time since diagnosis and whether patients had wAMD in one or two eyes; these comparisons were analyzed using either a two-sided Student's $t$-test (to compare mean values) or a two-tailed $Z$-test (to compare percentages). These analyses were based on the assumption of equal variance with a $5 \%$ significance level $(P<0.05)$. Tests were adjusted using the Bonferroni correction to counteract the problem of multiple and pairwise comparisons. Data analyses were performed in SPSS version 21 (IBM Corporation, Armonk, NY, USA), and all analyses were documented in syntax files.

\section{Results}

\section{Participants}

A total of 910 patients and 890 caregivers completed the questionnaire (Table 1$) ; 25.6 \%(n=233 / 910)$ of patients and $8.7 \%$ $(n=77 / 890)$ of caregivers $(n=77 / 890)$ were recruited by physician referral. The complete breakdown by country is shown in Table S1. The majority of patients had been diagnosed (74.7\%; $\mathrm{n}=680 / 910)$ and had been receiving anti-VEGF injections $(63.8 \% ; n=581 / 910)$ for more than 1 year, and many patients (82.1\%) also received support from a caregiver (Table 1). Most caregivers were nonprofessional $(93.5 \%$; $=832 / 890)$ (Table 1$)$. Many were related to the patient being a partner (23.3\%), child/ grandchild (47.3\%), or sibling (6.0\%) (Table 1).

\section{General impact of wAMD on patients and caregivers}

Figure 1 shows a snapshot of the overall effect of wAMD. The majority of patients $(71.6 \%)$ reported that wAMD had a negative impact on their life. The effect on the caregiver (unpaid/nonprofessional) was mixed, with $31.1 \%$ rating the
Table I Summary of patients and caregivers in the survey

\begin{tabular}{|c|c|}
\hline Patients $(n=9 \mid 0)$ & n (\%) \\
\hline \multicolumn{2}{|l|}{ wAMD diagnosis } \\
\hline One eye & $500(54.9)$ \\
\hline Two eyes & $410(45.1)$ \\
\hline \multicolumn{2}{|l|}{ Time since diagnosis } \\
\hline$\leq \mathrm{I}$ year & $230(25.3)$ \\
\hline$>\mathrm{I}-<3$ years & $420(46.2)$ \\
\hline$\geq 3-<6$ years & $203(22.3)$ \\
\hline$\geq 6$ years & $57(6.3)$ \\
\hline \multicolumn{2}{|l|}{ Duration of treatment } \\
\hline$\leq$ I year & $329(36.2)$ \\
\hline$>\mathrm{I}-<3$ years & $391(43.0)$ \\
\hline$\geq 3-<5$ years & $147(16.2)$ \\
\hline$\geq 5$ years & $43(4.7)$ \\
\hline \multicolumn{2}{|l|}{ Caregiver support received for daily tasks } \\
\hline Yes & $747(82.1)$ \\
\hline No & $163(17.9)$ \\
\hline \multicolumn{2}{|l|}{ Comorbid eye conditions ${ }^{\mathrm{a}}$} \\
\hline Cataracts & $73(18.6)$ \\
\hline Glaucoma & $42(10.7)$ \\
\hline DME & $49(12.5)$ \\
\hline Uveitis & I4 (3.6) \\
\hline Dry eye & $39(9.9)$ \\
\hline Long/short sighted & $107(27.3)$ \\
\hline Other & $56(14.3)$ \\
\hline None & $132(33.7)$ \\
\hline \multicolumn{2}{|l|}{ Caregivers $(n=890)$} \\
\hline \multicolumn{2}{|l|}{ Relationship } \\
\hline Partner & $207(23.3)$ \\
\hline Child/grandchild & $421(47.3)$ \\
\hline Sibling & $53(6.0)$ \\
\hline Professional caregiver & $58(6.5)$ \\
\hline Volunteer & $29(3.3)$ \\
\hline Neighbor/friend/other relative & $122(13.7)$ \\
\hline \multicolumn{2}{|l|}{ Cohabit with patient } \\
\hline Yes & $56 \mid(63.0)$ \\
\hline No & $329(37.0)$ \\
\hline \multicolumn{2}{|l|}{ Timing of support } \\
\hline Before diagnosis due to deteriorated vision & $296(33.3)$ \\
\hline Before diagnosis due to another health reason & I58 (17.8) \\
\hline Within a few weeks following diagnosis & $253(28.4)$ \\
\hline I-5 months after diagnosis & $99(11.1)$ \\
\hline $6-12$ months after diagnosis & $42(4.7)$ \\
\hline$>12$ months following diagnosis & $42(4.7)$ \\
\hline
\end{tabular}

Note: ${ }^{a}$ Question asked to patients $(n=392)$ who reported other health conditions. Abbreviations: DME, diabetic macular edema; wAMD, wet age-related macular degeneration.

impact of the patient's diagnosis as negative and $34.7 \%$ reporting that it had a positive impact (Figure 1A). The level of dependency of patients on caregivers after the wAMD diagnosis was rated as comparable between patients $(54.2 \%)$ and caregivers $(57.2 \%)$ (Figure 1B). Most patients required support for daily tasks 7 days per week $(28.0 \%)$ or on an "as-needed" basis (30.1\%) (Figure 1C). Patients with wAMD in one eye were significantly more likely to require support 

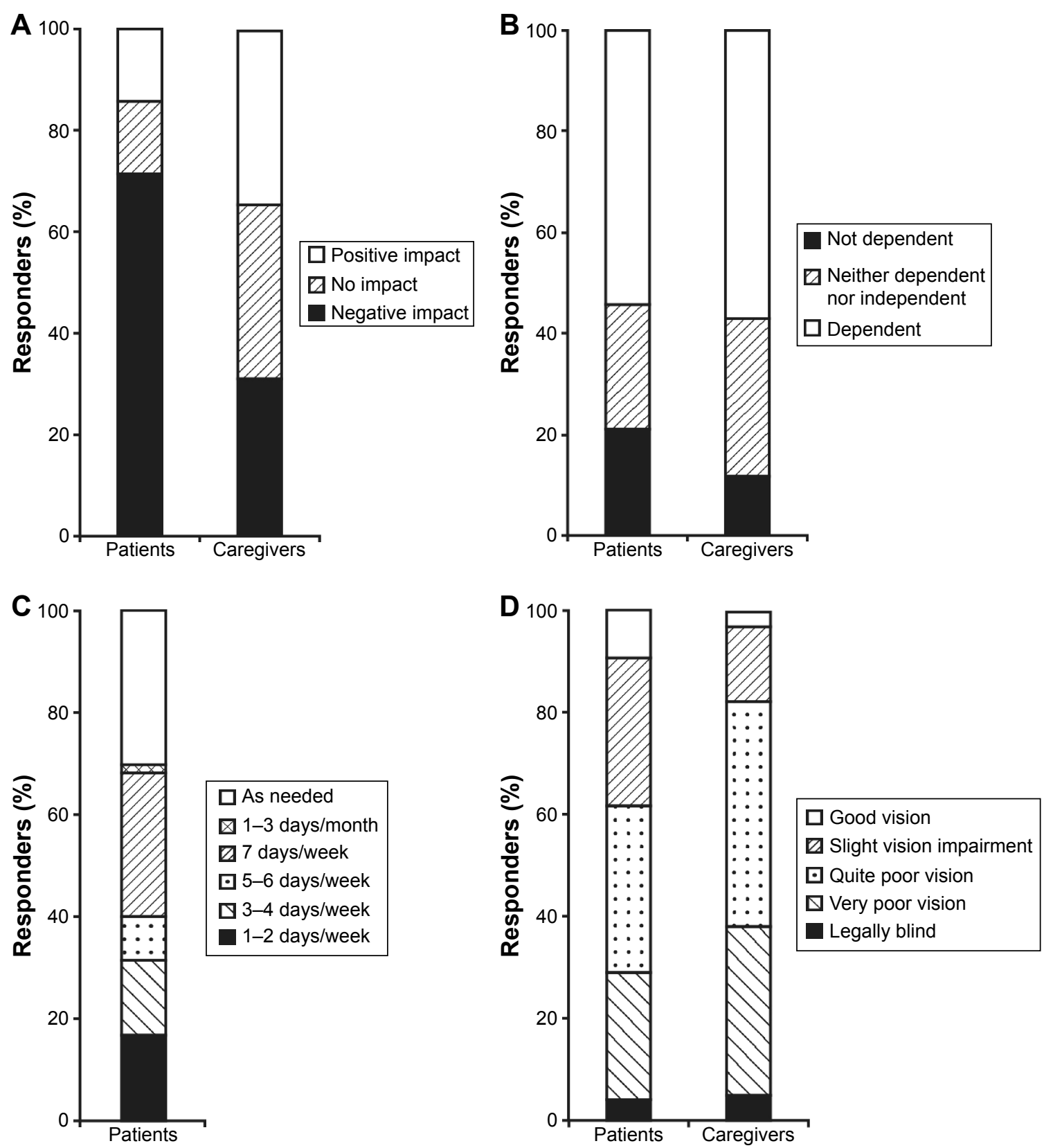

Figure I The effect of wAMD on patients and caregivers according to (A) overall impact, (B) dependence of patient on caregiver, (C) level of assistance required (patients only), and (D) rating of vision loss.

Notes: Questions asked for each effect: (A) What impact has wAMD had on your life (patients, $\mathrm{n}=910$ )? To what extent has becoming a caregiver for the patient had a negative or positive impact on your life (unpaid caregivers only, $n=832$ )? (B) Overall, how dependent are you on someone caring for you since your diagnosis on a scale of $\mathrm{I}-5$, where $\mathrm{I}$ is not at all dependent and 5 is extremely dependent (patients who have help with daily tasks, $n=747)$ ? Overall, how dependent is the patient since their diagnosis on a scale of $\mathrm{I}-5$, where $\mathrm{I}$ is not at all dependent and 5 is extremely dependent (caregivers, $\mathrm{n}=890$ )? (C) How often do you have a caregiver assist you (patients who have help with daily tasks, $\mathrm{n}=747$ )? (D) Currently, how would you rate your vision (patients, $\mathrm{n}=910$ )? Currently, how would you rate the patient's vision (caregivers, $\mathrm{n}=890$ )?

Abbreviation: WAMD, wet age-related macular degeneration.

on an "as-needed" basis (38.2\% vs $21.5 \% ; P<0.05)$. Overall, $57.6 \%$ of patients and $77.2 \%$ of caregivers rated patient vision as very poor or quite poor (Figure 1D).

\section{Emotional impact of wAMD on patients}

wAMD was associated with a range of negative emotions in patients, including fear (44.9\%), sadness $(39.9 \%)$, frustration (37.3\%), and depression (34.0\%) (Figure 2A).
Several negative emotions also increased with the time since diagnosis, including fear, frustration, and depression (Figure 2A). However, some patients also reported positive emotions such as hope (27.8\%), determination $(23.5 \%)$, and relief that it was not more serious (20.1\%), which did not change with time since diagnosis (Figure 2A). Despite $82.1 \%$ of patients receiving caregiver support, only $24.6 \%$ felt supported. 


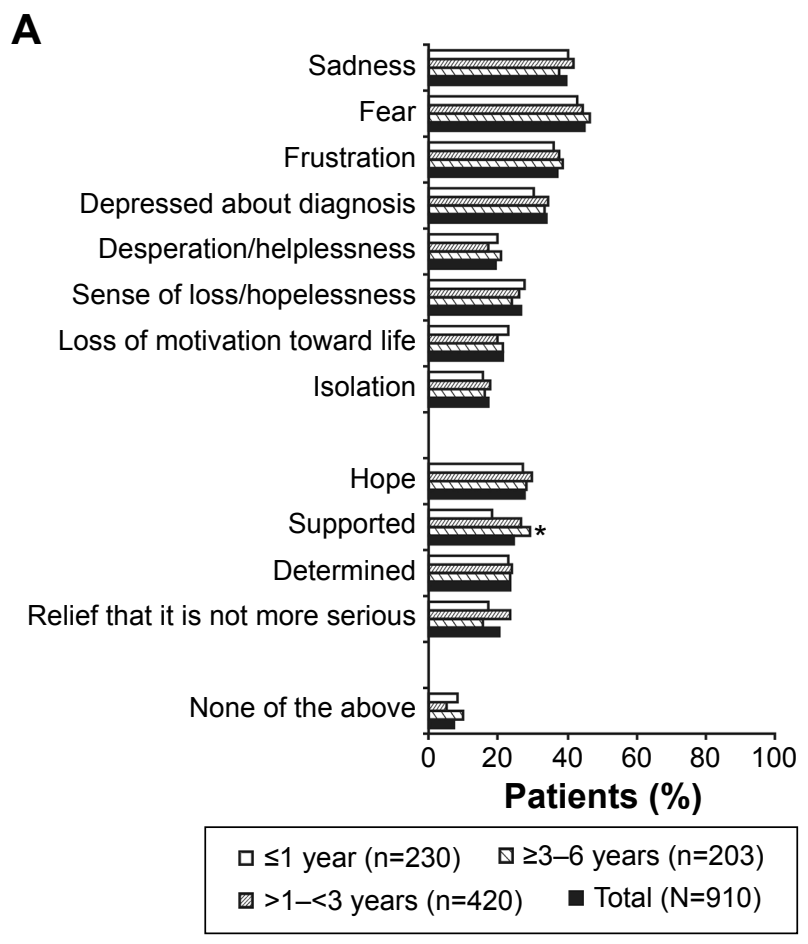

B

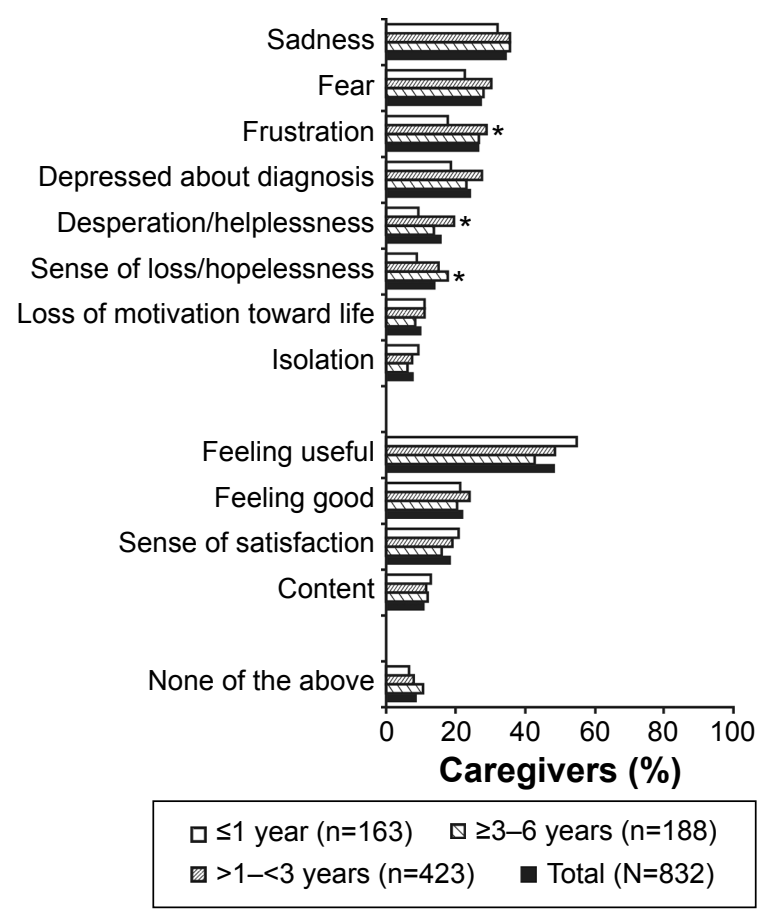

Figure 2 The emotional impact of wAMD on (A) patients and (B) caregivers (unpaid).

Notes: Data are presented as global and in subgroups according to time since diagnosis. $* P<0.05$ vs $\leq 1$ year. Questions asked for each effect: (A) What feelings have you experienced since your diagnosis with WAMD (patients)? (B) What feelings have you experienced since you started to help and support the patient (unpaid caregivers only)? Data not available for all patients/caregivers based on disease duration categories. Abbreviation: wAMD, wet age-related macular degeneration.

A number of negative emotions were greater in patients with wAMD in two eyes compared with one eye, including depression $(37.1 \%$ vs $31.4 \%$; $P=$ not significant, respectively), desperation/helplessness ( $24.1 \%$ vs $15.0 \% ; P<0.05$ ), and isolation ( $20.5 \%$ vs $14.6 \% ; P<0.05)$. Positive emotions were significantly greater in patients with wAMD in one eye, including relief that it was not more serious ( $22.8 \%$ vs $16.8 \%$; $P<0.05$ ), hope (32.6\% vs $22.0 \% ; P<0.05$ ), and determination $(28.4 \%$ vs $17.6 \% ; P<0.05)$. In patients who reported feeling fearful about their diagnosis, the greatest fear was permanent loss of vision $(60.0 \%$; $n=244 / 407)$.

\section{Emotional impact of wAMD on caregivers}

Similar to patients, caregivers commonly experienced sadness (34.9\%), fear (27.6\%), frustration (26.8\%), and depression (24.4\%) (Figure 2B). However, nearly half of all unpaid caregivers also felt useful (48.4\%). When caregivers were stratified according to length of time since patient diagnosis of wAMD ( $\leq 1$ year, $>1-<3$ years, $\geq 3-6$ years), a number of negative emotions were highlighted, which included frustration: $17.8 \%, 29.1 \% *$, and $26.6 \%$; depression about diagnosis: $18.4 \%, 27.4 \%$, and $22.9 \%$; loss/hopelessness: $8.6 \%, 14.9 \%$, and $17.6 \% *$; and desperation/helplessness: $9.2 \%, 19.6 \% *$, and $13.8 \%(* P<0.05$ vs $\leq 1$ year $)$, respectively. Positive emotions, such as feeling useful, decreased over the same timeframe (Figure 2B). In caregivers, negative (but not positive) emotions were also linked to whether the patient had wAMD in both eyes compared with one eye.

\section{Physical impact of wAMD on patients Day-to-day activities}

In patients, wAMD was associated with a number of negative disease consequences (Figure 3A). The most commonly reported disease consequences were difficulty reading $(61.1 \%)$, inability to drive (38.5\%), not engaging in the same activities as before (34.9\%), being uncomfortable leaving the house alone (34.8\%), and becoming less active (34.7\%). Many of the negative consequences of WAMD also increased with time since diagnosis. Patients with wAMD for $\geq 3-6$ years were significantly more likely to report difficulty reading compared with patients diagnosed for $\leq 1$ year (67.5\% vs $54.8 \% ; P<0.05$, respectively) (Figure $3 \mathrm{~A}$ ). Patients diagnosed for $\geq 3-6$ years were also significantly $(P<0.05)$ more likely to report a greater physical impact of wAMD, including an inability to engage in the same activities as before, having difficulty with stairs, cooking and facial recognition, and were uncomfortable leaving the house alone, when compared with patients diagnosed for $\leq 1$ year. 
A

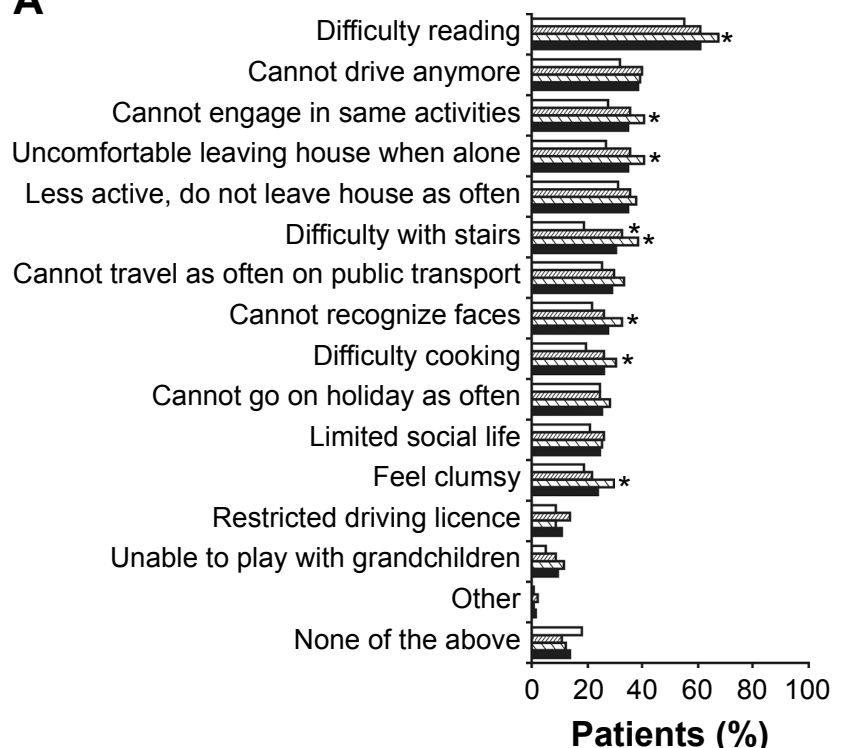

B

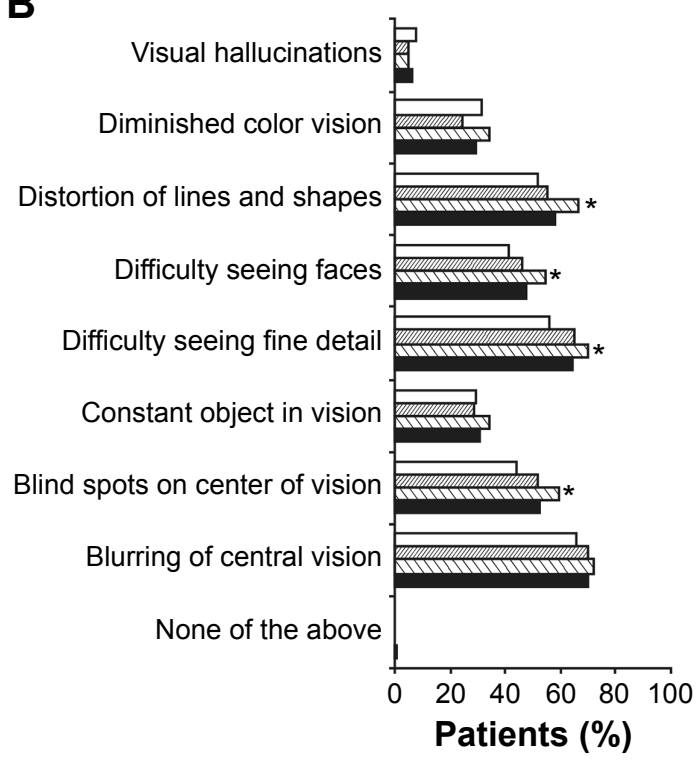

$\square \leq 1$ year $(n=230) \quad$ 口 $>1-<3$ years $(n=420) \quad \square \geq 3-6$ years $(n=203) \quad \square$ Total $(N=910)$

Figure 3 The physical impact of wAMD on patients based on (A) limitations in daily activities and (B) symptoms.

Notes: Data are presented as global and in subgroups according to time since diagnosis. $* P<0.05$ vs $\leq$ I year. Questions asked for each effect: (A) Since being diagnosed with wAMD which of the following statements apply to you (patients)? (B) What kind of problems do you currently experience or have previously experienced with your vision (patients)? Data not available for all patients based on disease duration categories.

Abbreviation: wAMD, wet age-related macular degeneration.

The physical impact of wAMD was also greater in patients with both eyes affected compared with one eye; this was particularly notable for inability to drive $(44.4 \%$ vs $33.6 \%$; $P<0.05$, respectively), lack of facial recognition (37.1\% vs $20.4 \% ; P<0.05)$, difficulty using stairs $(34.1 \%$ vs $28.0 \%$; $P<0.05)$, reduced travel on public transportation $(33.9 \%$ vs $25.4 \%$; $P<0.05)$, reduction in holidays $(30.0 \%$ vs $22.2 \%$; $P<0.05)$, limited social life (27.8\% vs $22.0 \% ; P<0.05)$, feeling clumsy ( $27.6 \%$ vs $20.6 \% ; P<0.05$ ), and inability to play with grandchildren $(12.9 \%$ vs $6.0 \% ; P<0.05)$.

\section{Falls and accidents}

A number of patients reported a fall (25.7\%) or accident $(7.3 \%)$ in the previous 2 years due to vision impairment associated with wAMD, and $34.2 \%$ of these patients had been hospitalized as a result of a fall. The number of falls increased with time since diagnosis: $22.2 \%(\leq 1$ year since diagnosis), $25.0 \%$ ( $>1-<3$ years), and $28.1 \%$ ( $\geq 3-6$ years). However, the number of accidents (such as car accidents) was higher in those with a more recent diagnosis: $8.3 \%$ ( $\leq 1$ year), $8.3 \%$ ( $>1-<3$ years), and $3.0 \%$ ( $\geq 3-6$ years). The number of falls and accidents was significantly greater in patients with wAMD in two eyes compared with one eye $(32.2 \%$ vs $20.4 \% ; P<0.05$ [falls] and $11.5 \%$ vs $3.8 \%$; $P<0.05$ [accidents]).

\section{Troublesome symptoms}

The main symptoms of wAMD reported by patients are summarized in Figure 3B. These include blurring of central vision (69.7\%), difficulty seeing fine detail (64.1\%), and distortion of lines and shapes (58.2\%). Many symptoms increased with time since diagnosis, and blind spots in center of vision, difficulty seeing fine detail and faces, and distortion of lines and shapes were all significantly $(P<0.05)$ greater in patients diagnosed between $\geq 3$ and 6 years compared with $\leq 1$ year (Figure 3B). A number of symptoms were also reported with significantly more frequency in patients with wAMD in both eyes compared with one eye, including blurring of central vision $(74.4 \%$ vs $65.8 \%$; $P<0.05)$, constant object in vision (35.6\% vs $26.4 \%$; $P<0.05)$, difficulty seeing faces ( $53.7 \%$ vs $42.0 \% ; P<0.05$ ), and visual hallucinations $(9.8 \%$ vs $3.0 \% ; P<0.05)$.

\section{Physical impact of wAMD on caregivers Day-to-day activities}

The role of the caregiver (as rated by the caregiver and patient) is summarized in Figure 4A. The most frequent support provided by the caregiver was associated with arranging travel (85.5\% [caregiver]). The provision of support was rated as greater by the caregiver than the patient; this was greatest for help with health care professional (HCP) discussions (including treatment plan) (65.5\% [caregivers] 


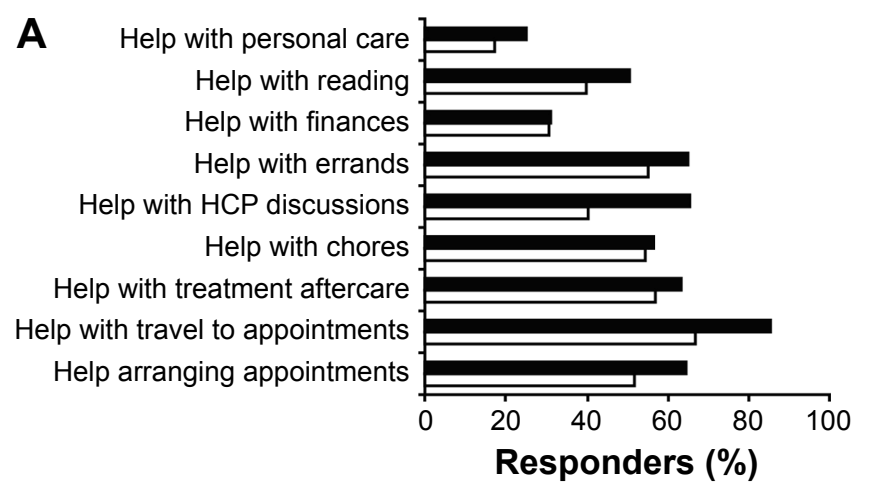

口 Caregivers $\square$ Patients

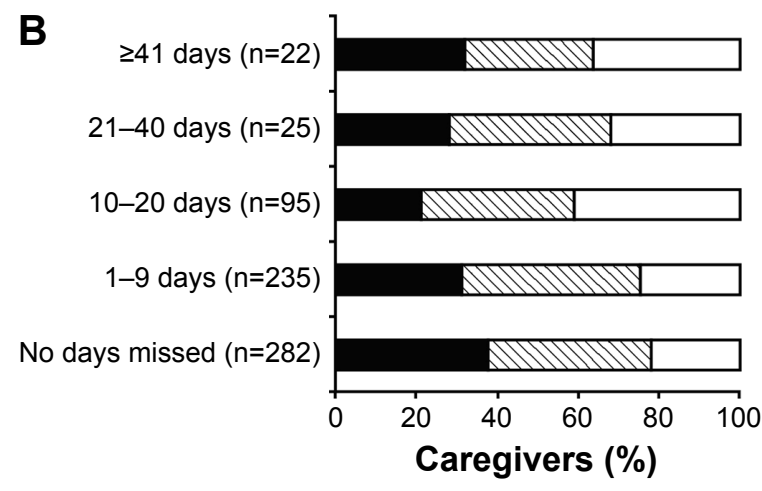

Dot inconvenient $\square$ Neither convenient $\square$ Inconvenient nor inconvenient

Figure 4 The physical impact of wAMD on caregivers based on $(\mathbf{A})$ the role of the caregiver and $(\mathbf{B})$ convenience of patient's current treatment according to the number of employment days/personal obligations the caregiver has missed.

Notes: Questions asked for each effect: (A) In what ways does the caregiver help you (patients who have help with daily tasks, $\mathrm{n}=747)$ ? In what ways do you help the patients (caregivers, $n=890$ )? (B) Based on the following questions: Approximately how many days of employment or personal obligations have you had to miss over the last 12 months because you were helping the patient in some way (unpaid, employed caregivers only, $\mathrm{n}=659$ )? To what extent do you find the patient's current treatment an inconvenience (caregivers, $n=890$ )? Treatment includes traveling to and from appointments and helping the patients to manage their condition (physically and emotionally). Abbreviations: HCP, health care professional; wAMD, wet age-related macular degeneration.

vs $40.4 \%$ [patients]). The level of support provided by caregivers tended to increase with duration of diagnosis. Help with treatment aftercare was provided by $54.9 \%$ of caregivers for patients diagnosed within $\leq 1$ year and by $64.8 \%$ of caregivers for patients diagnosed between $\geq 3$ and 6 years. Help with shopping increased from $53.7 \%$ to $64.8 \%$, and help with reading increased from $39.4 \%$ to $57.8 \%$.

\section{Productivity}

Overall, 27.2\% ( $n=242 / 890)$ of caregivers rated the patient's current treatment (ie, caregiving) as inconvenient. The level of inconvenience was associated with the number of days of employment/personal obligations missed (Figure 4B). In total, $57.2 \%(n=377 / 659)$ of caregivers missed days of employment/personal obligations during the previous 12 months as a result of helping the patient; $14.4 \%$ ( $n=95 / 659)$ missed 10-20 days of employment/personal obligations, with $41.0 \%$ of these caregivers rating the patient's treatment (ie, caregiving) as inconvenient.

\section{Discussion}

This global survey showed that wAMD has a major emotional and physical impact on the lives of patients and caregivers, and may be associated with an economic burden (ie, lost caregiver productivity due to missed workdays). The majority of patients had been diagnosed for more than 1 year, and most patients experienced a range of problems. Many of these impairments were significantly worse in patients who had wAMD in both eyes, and in those with a longer duration of disease (notably depression).
The high rate of depressive symptoms observed in patients in our survey is consistent with other published estimates, which range from $30 \%$ to $42 \%,{ }^{2,11}$ and is two to three times higher than estimates in an elderly community-dwelling population without wAMD (which was approximately $8 \%-20 \%) .{ }^{12}$ wAMD has also been shown to double the fall rate (from $8 \%$ to $16 \%$ ) and quadruple the need for daily assistance (from $7 \%$ to $29 \%$ ) compared with controls. ${ }^{11}$ Other reports also highlight that emotional problems increase in patients with bilateral wAMD. ${ }^{13}$ However, a major impact on day-to-day activities was also observed for patients with wAMD in one eye alone in our survey. Another study in 86 elderly adults found that emotional distress was significantly greater in patients with wAMD blindness in one eye only. ${ }^{5}$ A possible explanation for this may be related to uncertainty surrounding further vision loss. ${ }^{14}$

The current survey also showed that wAMD had a major impact on nonprofessional (unpaid) caregivers. Despite $48.4 \%$ of caregivers having reported feeling useful, many also reported feeling sad, fearful, and frustrated, with these emotions increasing with duration of the patient's diagnosis and their disease severity (two eyes vs one eye). It was interesting to note that $24.4 \%$ of caregivers $(33.6 \%$ of caregivers supporting a patient with wAMD in both eyes) experienced depression about the diagnosis. In general, the emotional impact on caregivers was comparable with that experienced by the patient. Although the support provided by the caregiver was mostly practical help with day-to-day activities, around one-quarter expressed that the patient's treatment (ie, caregiving) was inconvenient. Many caregivers had missed days 
of employment/personal obligations as a result of supporting the patient. In the survey of 500 caregivers in Australia, which used a reconfigured version of this questionnaire, one-third of caregivers reported high dependence by patients, $53.3 \%$ of caregivers provided daily care to patients who were blind, but $80.6 \%$ still provided "as-needed" support to those patients whose vision was rated as good. ${ }^{10}$

The findings show that despite receiving anti-VEGF agents, which can improve or stabilize vision, many patients with wAMD continue to suffer adverse affective and functional consequences. It is possible that a number of other factors, which were not explored in detail in this survey, could be considered for reducing the negative impact on patients and caregivers, including tailoring of treatment and follow-up visits. The survey also reports that $42.3 \%$ of patients attended clinic visits every 4-6 weeks, with $42.5 \%$ having treatment at every visit (data not shown). This suggests that many patients may have been undertreated. A number of observational studies have found that the frequency of actual anti-VEGF injections (such as monthly ranibizumab or bevacizumab) is lower than that recommended in large-scale studies, and according to the label. ${ }^{15,16}$ In the AURA (a retrospective non-interventional study to assess the effectiveness of existing anti-VEGF treatment regimens in patients with wet age-related macular degeneration) study, 2,227 wAMD patients were followed-up for a 2 -year period. ${ }^{17}$ During this time, patients received a mean of 5.0 anti-VEGF injections (mainly ranibizumab) in year 1 and a mean of 2.2 injections in year 2; this was coupled with a gradual decline in visual acuity. Unfortunately, no studies have explored the effect of different dosing regimens and follow-up schedules on patient and caregiver outcomes, including depression.

Although the survey is valuable in providing an overview of the impact of wAMD on patients and caregivers, it does have a number of limitations inherent to the observational design. The questionnaire was devised by experts to cover all aspects of wAMD, but it is not validated and is still subjective, and some questions (including the definition of depression) may be perceived differently by responders from different countries. We did not correlate the severity of visual acuity impairment at baseline on emotional and physical outcomes addressed by the questionnaire. The impact of other baseline characteristics was were also not assessed, and this could have influenced some of the participants' answers, especially for patients suffering with depression or anxiety before the wAMD diagnosis, resulting in bias. It is also not possible to distinguish the severity of the outcomes reported, or to gauge the level of symptoms such as depres- sion. Furthermore, the results are based on pooled findings from nine countries, and the impact of wAMD varied between countries (Table S2). Notably, patients who reported difficulty reading ranged from $38.8 \%$ (UK) to $79.0 \%$ (Brazil), and patients who could not drive anymore ranged from $10.9 \%$ (Japan) to 48.4\% (Australia). The level of support for dayto-day tasks also varied between countries (Table S3). Help with reading (caregiver perspective) ranged from $23.1 \%$ (Japan) to $79.2 \%$ (Spain). It was interesting to note that the level of support provided was, in general, rated as higher by caregivers than patients across all countries.

\section{Conclusion}

In summary, the findings from this survey indicate that there is a range of emotional and physical problems associated with wAMD in patients, including vision-related depression, mobility, and limitations in day-to-day activities. The impact on nonprofessional caregivers may also be underestimated in terms of emotional impact (such as depression) and loss of productivity. However, it is interesting to note that $82.1 \%$ of patients received caregiver support, but only $24.6 \%$ felt supported - this highlights a key issue. Previous reports suggest that HCPs may underestimate the impact of wAMD. ${ }^{18}$ These findings also highlight the need for a more holistic approach to the problems encountered by wAMD patients. In a previous study of 477 patients with moderate-to-severe vision loss (43\% had wAMD), visual acuity, presence of comorbidities, and dependence on family and friends emerged as the strongest independent predictors of greater rehabilitation needs. ${ }^{19}$ Depending on the health care system, the treating HCP could address some of these issues in more detail, ensuring adequate diagnosis and treatment of comorbidities, including depression, other eye disorders, and age-related disorders, or referral to other HCPs where necessary, and this wider approach may help to reduce the burden of wAMD in the longer term. The burden on the caregiver also highlights the need for a wider approach to service provision, support, and psychological resources, which is consistent with a previous meta-synthesis of qualitative research in this area. ${ }^{6}$

\section{Acknowledgment}

These data have been presented at the Royal College of Ophthalmologists' Congress (May 20-22, 2014, Birmingham, UK), the German Retina Society Congress (June 13-14, 2014, Düsseldorf, Germany), and the International Society for Pharmacoeconomics and Outcomes Research (November 8-12, 2014, Amsterdam, the Netherlands). The contribution of the Fondazione GB Bietti-IRCCS to this paper was sup- 
ported by the Italian Ministry of Health and by Fondazione Roma. The survey was sponsored by Bayer HealthCare Pharmaceuticals. Medical writing assistance was provided by PAREXEL and was funded by Bayer HealthCare Pharmaceuticals.

\section{Disclosure}

Monica Varano has served on advisory boards for Bayer, Novartis, and Allergan. Nicole Eter, Steve Winyard, and Rafael Navarro report no conflicts of interest in this study. Kim U Wittrup-Jensen is an employee of Bayer Pharma AG. Julie Heraghty is a CEO of Macular Disease Foundation Australia, which receives funding from Bayer and Novartis. The authors resport no other conflicts of interest in this work.

\section{References}

1. Wong WL, Su X, Li X, et al. Global prevalence of age-related macular degeneration and disease burden projection for 2020 and 2040: a systematic review and meta-analysis. Lancet Glob Health. 2014;2(2):e106-e116.

2. Casten RJ, Rovner BW, Tasman W. Age-related macular degeneration and depression: a review of recent research. Curr Opin Ophthalmol. 2004;15(3):181-183.

3. Casten RJ, Rovner BW. Update on depression and age-related macular degeneration. Curr Opin Ophthalmol. 2013;24(3):239-243.

4. Hassell JB, Lamoureux EL, Keeffe JE. Impact of age related macular degeneration on quality of life. Br J Ophthalmol. 2006;90(5): 593-596.

5. Williams RA, Brody BL, Thomas RG, Kaplan RM, Brown SI. The psychosocial impact of macular degeneration. Arch Ophthalmol. 1998;116(4): 514-520.

6. Bennion AE, Shaw RL, Gibson JM. What do we know about the experience of age related macular degeneration? A systematic review and meta-synthesis of qualitative research. Social Sci Med. 2012;75(6): 976-985.

7. Bandello F, Lafuma A, Berdeaux G. Public health impact of neovascular age-related macular degeneration treatments extrapolated from visual acuity. Investig Ophthalmol Visual Sci. 2007;48(1):96-103.
8. Pinquart M, Sorensen S. Associations of stressors and uplifts of caregiving with caregiver burden and depressive mood: a meta-analysis. J Gerontol B Psychol Sci Soc Sci. 2003;58(2):112-128.

9. Robison J, Fortinsky R, Kleppinger A, Shugrue N, Porter M. A broader view of family caregiving: effects of caregiving and caregiver conditions on depressive symptoms, health, work, and social isolation. J Gerontol B Psychol Sci Soc Sci. 2009;64(6):788-798.

10. Gopinath B, Kifley A, Cummins R, Heraghty J, Mitchell P. Predictors of psychological distress in caregivers of older persons with wet age-related macular degeneration. Aging Ment Health. 2015;19(3):239-246.

11. Soubrane G, Cruess A, Lotery A, et al. Burden and health care resource utilization in neovascular age-related macular degeneration: findings of a multicountry study. Arch Ophthalmol. 2007;125(9):1249-1254.

12. World Health Organization. World Health Report: Mental Health: New Understanding, New Hope. Geneva: World Health Organization; 2001. Available from: http://www.who.int/whr/2001/en/whr01_en.pdf. Accessed October 7, 2014

13. Berman K, Brodaty H. Psychosocial effects of age-related macular degeneration. Int Psychogeriatr. 2006;18(3):415-428.

14. Slakter JS, Stur M. Quality of life in patients with age-related macular degeneration: impact of the condition and benefits of treatment. Surv Ophthalmol. 2005;50(3):263-273.

15. Holekamp NM, Liu Y, Yeh WS, et al. Clinical utilization of anti-VEGF agents and disease monitoring in neovascular age-related macular degeneration. Am J Ophthalmol. 2014;157(4):825-833.

16. Lad EM, Hammill BG, Qualls LG, Wang F, Cousins SW, Curtis LH. Anti-VEGF treatment patterns for neovascular age-related macular degeneration among medicare beneficiaries. Am J Ophthalmol. 2014; 158(3):537-543

17. Holz FG, Tadayoni R, Beatty S, et al. Multi-country real-life experience of anti-vascular endothelial growth factor therapy for wet age-related macular degeneration. Br J Ophthalmol. 2015;99(2):220-226.

18. Stein JD, Brown MM, Brown GC, Hollands H, Sharma S. Quality of life with macular degeneration: perceptions of patients, clinicians, and community members. Br J Ophthalmol. 2003;87(1):8-12.

19. O'Connor PM, Lamoureux EL, Keeffe JE. Predicting the need for low vision rehabilitation services. Br J Ophthalmol. 2008;92(2):252-255. 


\section{Supplementary materials}

Table SI Patient $(n=9 \mid 0)$ and caregiver $(n=890)$ recruitment by country $(n)$

\begin{tabular}{|c|c|c|c|c|c|c|}
\hline \multirow[t]{2}{*}{ Country } & \multirow{2}{*}{$\frac{\text { Patients }}{\text { Total }}$} & \multicolumn{2}{|c|}{ Recruitment from referrals } & \multirow{2}{*}{$\frac{\text { Caregivers }}{\text { Total }}$} & \multicolumn{2}{|c|}{ Recruitment from referrals } \\
\hline & & $\begin{array}{l}\text { Not from } \\
\text { physician referral }\end{array}$ & $\begin{array}{l}\text { From physician } \\
\text { referral }\end{array}$ & & $\begin{array}{l}\text { Not from } \\
\text { physician referral }\end{array}$ & $\begin{array}{l}\text { From physician } \\
\text { referral }\end{array}$ \\
\hline Australia & 122 & 122 & 0 & 107 & 107 & 0 \\
\hline Canada & 111 & 110 & I & 106 & 92 & 14 \\
\hline Germany & 112 & 103 & 9 & 105 & 105 & 0 \\
\hline Spain & 103 & 72 & 31 & 101 & 101 & 0 \\
\hline France & 102 & 42 & 60 & 105 & 103 & 2 \\
\hline UK & 103 & 83 & 20 & 107 & 107 & 0 \\
\hline Italy & 102 & 52 & 50 & 107 & 105 & 2 \\
\hline Japan & 55 & 55 & 0 & 52 & 52 & 0 \\
\hline Brazil & 100 & 38 & 62 & 100 & 41 & 59 \\
\hline
\end{tabular}

Table S2 The impact (\%) of wAMD on a patient's ability to read or drive as rated by patients $(\mathrm{n}=910)$ and caregivers $(\mathrm{n}=890)$ in each country

\begin{tabular}{|c|c|c|c|c|c|c|c|c|c|}
\hline Country & Germany & Spain & France & Italy & UK & Australia & Brazil & Canada & Japan \\
\hline Patients, n & 112 & 103 & 102 & 102 & 103 & 122 & 100 & 111 & 55 \\
\hline Difficulty reading, \% & 60.7 & 72.8 & 60.8 & 72.5 & 38.8 & 62.3 & 79.0 & 54.1 & 40.0 \\
\hline Cannot drive anymore, $\%$ & 37.5 & 42.7 & 30.4 & 45.1 & 35.0 & 48.4 & 45.0 & 36.9 & 10.9 \\
\hline Caregivers, n & 105 & 101 & 105 & 107 & 107 & 107 & 100 & 106 & 52 \\
\hline Difficulty reading, \% & 69.5 & 77.2 & 65.7 & 72.9 & 58.9 & 71.0 & 84.0 & 66.0 & 44.2 \\
\hline Cannot drive anymore, $\%$ & 68.6 & 42.6 & 61.0 & 48.6 & 55.1 & 44.9 & 63.0 & 61.3 & 30.8 \\
\hline
\end{tabular}

Abbreviation: wAMD, wet age-related macular degeneration.

Table S3 The level of support (\%) given by caregivers for daily tasks as rated by patients $\left(\mathrm{n}=747^{*}\right)$ and caregivers $(\mathrm{n}=890)$ in each country

\begin{tabular}{|c|c|c|c|c|c|c|c|c|c|}
\hline Country & Germany & Spain & France & Italy & UK & Australia & Brazil & Canada & Japan \\
\hline Patients, $\mathbf{n}$ & 100 & 97 & 61 & 95 & 81 & 96 & 97 & 79 & 41 \\
\hline Help with personal care, \% & 4.0 & 28.9 & 14.8 & 14.7 & 12.3 & 17.7 & 40.2 & 7.6 & 4.9 \\
\hline Help with reading, $\%$ & 27.0 & 60.8 & 41.0 & 34.7 & 19.8 & 57.3 & 62.9 & 22.8 & 9.8 \\
\hline Help with finances, \% & 32.0 & 30.9 & 24.6 & 36.8 & 23.5 & 21.9 & 49.5 & 31.6 & 9.8 \\
\hline Help with errands, \% & 46.0 & 51.5 & 50.8 & 50.5 & 39.5 & 78.1 & 74.2 & 53.2 & 39.0 \\
\hline Help with HCP discussions, \% & 34.0 & 47.4 & 42.6 & 37.9 & 23.5 & 45.8 & 63.9 & 36.7 & 14.6 \\
\hline Help with chores, \% & 50.0 & 48.5 & 55.7 & 41.1 & 37.0 & 72.9 & 69.1 & 70.9 & 34.1 \\
\hline Help with treatment aftercare, $\%$ & 50.0 & 62.9 & 50.8 & 65.3 & 32.1 & 65.6 & 80.4 & 55.7 & 26.8 \\
\hline Help with travel to appointments, \% & 56.0 & 74.2 & 50.8 & 83.2 & 51.9 & 70.8 & 80.4 & 65.8 & 53.7 \\
\hline Help arranging appointments, \% & 43.0 & 40.2 & 41.0 & 50.5 & 59.3 & 64.6 & 79.4 & 49.4 & 14.6 \\
\hline Caregivers, $\mathbf{n}$ & 105 & 101 & 105 & 107 & 107 & 107 & 100 & 106 & 52 \\
\hline Help with personal care, \% & 20.0 & 38.6 & 26.7 & 22.4 & 19.6 & 19.6 & 40.0 & 19.8 & 15.4 \\
\hline Help with reading, \% & 42.9 & 79.2 & 54.3 & 36.4 & 41.1 & 55.1 & 60.0 & 50.9 & 23.1 \\
\hline Help with finances, \% & 37.1 & 34.7 & 28.6 & 13.1 & 37.4 & 28.0 & 46.0 & 30.2 & 21.2 \\
\hline Help with errands, \% & 67.6 & 62.4 & 66.7 & 51.4 & 63.6 & 81.3 & 64.0 & 69.8 & 51.9 \\
\hline Help with HCP discussions, \% & 69.5 & 70.3 & 53.3 & 58.9 & 57.9 & 94.4 & 70.0 & 64.2 & 36.5 \\
\hline Help with chores, \% & 71.4 & 51.5 & 47.6 & 43.9 & 55.1 & 59.8 & 61.0 & 59.4 & 63.5 \\
\hline Help with treatment aftercare, \% & 64.8 & 72.3 & 52.4 & 65.4 & 60.7 & 62.6 & 76.0 & 64.2 & 42.3 \\
\hline Help with travel to appointments, \% & 89.5 & 89.1 & 77.1 & 81.3 & 86.0 & 96.3 & 91.0 & 78.3 & 76.9 \\
\hline Help arranging appointments, \% & 72.4 & 46.5 & 54.3 & 59.8 & 62.6 & 80.4 & 88.0 & 67.0 & 36.5 \\
\hline
\end{tabular}

Note: *Only those who have help with daily tasks.

Abbreviation: HCP, health care professional. 


\section{Publish your work in this journal}

Clinical Ophthalmology is an international, peer-reviewed journal covering all subspecialties within ophthalmology. Key topics include: Optometry; Visual science; Pharmacology and drug therapy in eye diseases; Basic Sciences; Primary and Secondary eye care; Patien Safety and Quality of Care Improvements. This journal is indexed on Submit your manuscript here: http://www.dovepress.com/clinical-ophthalmology-journal
Dovepress

PubMed Central and CAS, and is the official journal of The Society of Clinical Ophthalmology (SCO). The manuscript management system is completely online and includes a very quick and fair peer-review system, which is all easy to use. Visit http://www.dovepress.com/ testimonials.php to read real quotes from published authors. 